\title{
A PROPOSAL FOR THE SURFACE ROUGHNESS WAKE FIELD MEASUREMENT AT THE TESLA TEST FACILITY
}

\author{
A. Novokhatski, M. Timm *, T. Weiland, \\ TEMF, TU-Darmstadt, Schlossgartenstr. 8, D-64289 Darmstadt, Germany \\ H. Schlarb, DESY, Nottkestr. 85, D-22603 Hamburg, Germany
}

\begin{abstract}
The wake fields due to the rough surface of the vacuum chamber have a major influence on the beam dynamics in Linear Colliders and Free Electron Lasers. These wake fields mainly consists of the fundamental tube mode, modified by the rough boundary condition, which decreases its phase velocity to the speed of light. Its wavelength is proportional to the square root of the tube radius and the roughness depth and is comparable to the FEL bunch length, i.e. in the order of 10-30 micron. To study this effect with the beam available at the TESLA Test Facility (TTF) the use of tubes with an artificially increased roughness is suggested. The expected pulse power of these wakes, caused by the 250 micron bunch carrying a charge of $1 \mathrm{nC}$, can reach up to several MW. In this paper a preliminary design of an installation of a surface roughness wake field experiment at the TESLA Test Facility is presented.
\end{abstract}

\section{INTRODUCTION}

The conservation of a small emittance is a major requirement for the feasibility of the experiments in Linear Colliders, especially for the FEL. As the bunch length becomes very short, the contribution of the surface roughness wake fields is not negligible anymore. The longitudinal wake fields increase the energy spread, while the transverse dipole wake fields increase the bunch tails offset from the axis.

But even for longer bunches, in synchrotrons or damping rings, the roughness wake fields might have an influence on beam dynamics. They are suspected to cause sawtooth instabilities [4].

However the model used do describe the surface roughness wake field effect $[1,2,3]$ has to be verified by measurements.

\section{SURFACE ROUGHNESS WAKE FIELDS}

Due to the small corrugations at the beam pipe the fundamental mode in the tube is slightly modified. By this means its phase velocity is decreased to the speed of light at a very high frequency. Its group velocity is very close to the speed of light but does not reach it. No mode with a higher radial dependency experiences the same [3]. The bunch is excit-

\footnotetext{
*Email: timm@temf.tu-darmstadt.de
}

ing this mode only. Thus the wake field consists of one single mode running synchronous with the bunch (Fig 1).

The model of a thin dielectric layer can be employed to estimate the wake fields [1].

In the monopole case $(m=0)$ an estimation for the wave number is

$$
k_{0}^{2}=f \cdot \frac{4}{a \delta}
$$

where $a$ the tube radius and $\delta$ the rms thickness of the surface roughness. The factor $f$ reflects the influence of the roughness shape and its size in comparison to the wave length of the rough tube mode. It is in the order of 1 . These parameters mainly influence the frequency. It does not depend on any periodicity of the roughness, if the number of roughness bumps per wavelength of the rough tube mode is sufficiently large. The longitudinal wake function is

$$
w_{0}^{\|}(s)=\frac{Z_{0} c}{\pi a^{2}} \cos \left(k_{0} s\right) \cdot e^{-\alpha(l) s}
$$

using the impedance of free space $Z_{0}=\sqrt{\mu_{0} / \varepsilon_{0}} . c$ denotes the speed of light. The attenuation $\alpha$ is a function of the tube length $l$ and of the roughness shape. The wake amplitude is inversely proportional to the waveguides cross-section The dipole case can be described similarly. The dipole wake field has the same frequency as the monopole wake field. Its amplitude is twice as large as in the monopole case [2].

\subsection{Development of the Wake Field Pulse}

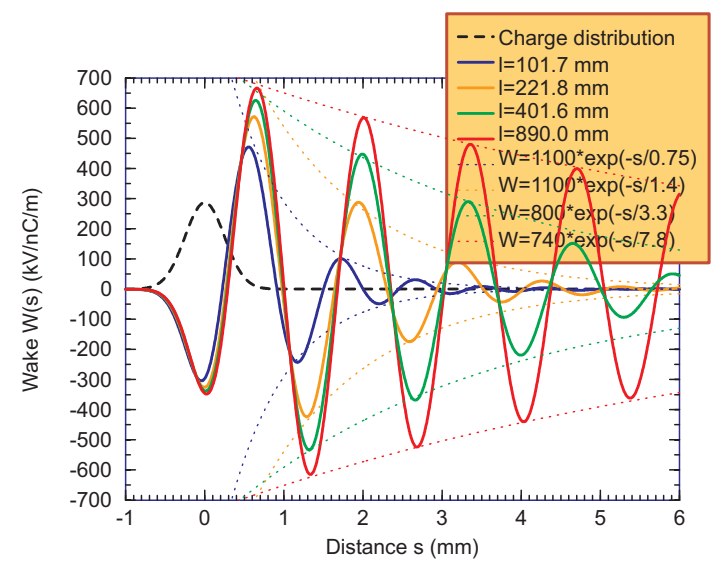

Figure 1: Time development of the the rf-wake field pulse created by a $250 \mu \mathrm{m}$ bunch in a tube with $5 \mathrm{~mm}$ radius and an rms roughness depth of $50 \mu \mathrm{m}$. 
The normalized wake amplitude stays constant inside the Bunch. The rf-pulse length is increasing proportional to the tube length. Accordingly the pulse power of a roughness wake pulse in a given tube is constant too, while its average power is increasing. The attenuation is inversely proportional to the tube length (Fig. 1).

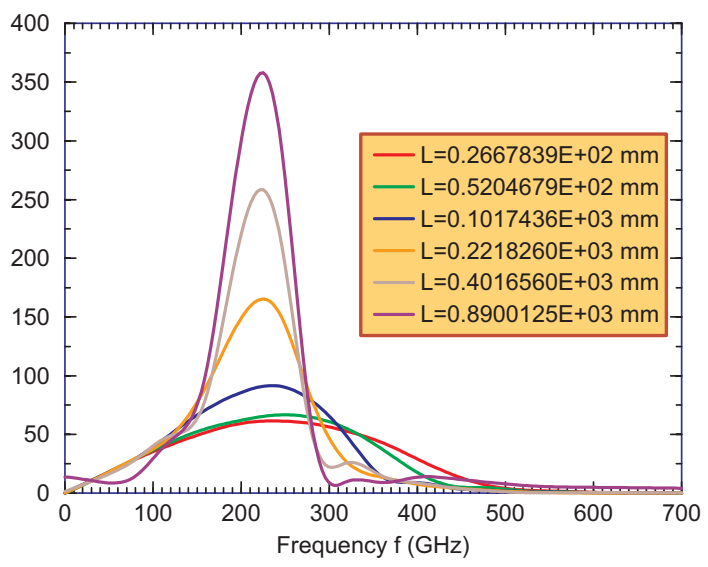

Figure 2: Time development of the spectrum of the rf-wake field pulse created by a $250 \mu \mathrm{m}$ bunch in a tube with $5 \mathrm{~mm}$ radius and an rms roughness depth of $50 \mu \mathrm{m}$.

The bandwidth of the rf-pulse is decreasing with the tube length. Fig 2 shows the development of the frequency spectrum.

\subsection{Normalized Description}

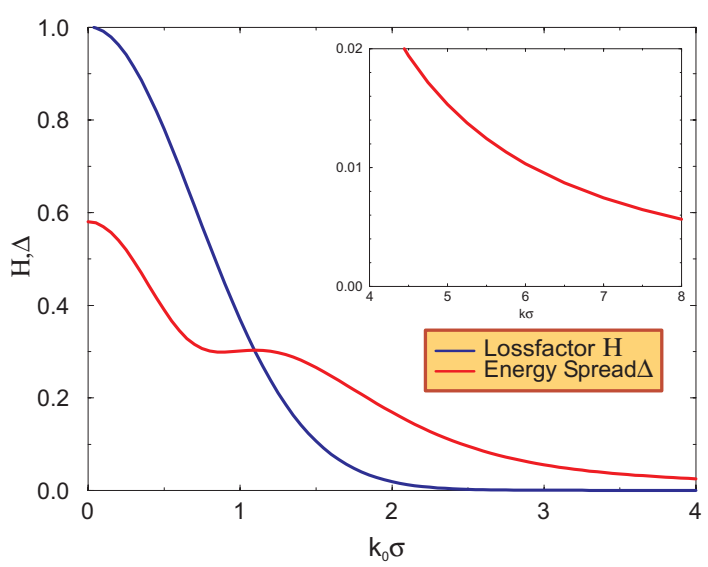

Figure 3: Loss factor $\mathrm{H}$ and energy spread $\Delta$ due to the rough tube mode in normalized description. [3]:

Introducing a normalized description, the loss factor is

$$
\mathrm{H}\left(k_{0} \sigma_{z}\right)=e^{-\left(k_{0} \sigma_{z}\right)^{2}} .
$$

It gives the energy transported by the rf-wave traveling along the tube. The normalized energy spread is:

$\Delta^{2}\left(k_{0} \sigma_{z}\right)=\frac{1}{\sqrt{2 \pi}} \int_{-\infty}^{\infty} W_{0}^{\|^{2}}\left(k_{0} \sigma_{z} z\right) e^{-\frac{z^{2}}{2}} d z-e^{-2\left(k_{0} \sigma_{z}\right)^{2}}$
Fig. 3 shows the normalized energy spread and loss factor. The wave number $k_{0}$ is a property of the beam pipe only, within the range of validity of the model, i.e. the bunch length has to be larger than the average distance between roughness peaks. For larger values of $k_{0} \sigma_{z}$ the loss factor becomes rather small, but the energy spread is still not negligible.

\subsection{Wake Fields in Waveguides with Different Apertures}

The loss factor and the energy spread of a Gaussian bunch in a cylindrical, rough waveguide are

$$
k_{\text {loss }}=\frac{Z_{0} c}{2 \pi a^{2}} \mathrm{H}\left(k_{0} \sigma_{z}\right), \text { and } \quad \Delta E=\frac{Z_{0} c}{2 \pi a^{2}} \Delta\left(k_{0} \sigma_{z}\right)
$$

They are inversely proportional to the cross-section of the wave guide. In case of an elliptical waveguide the wave number and the loss factor are

$$
k_{0}^{2}=f \frac{2}{\delta} \frac{a+b}{a b}, \quad k_{\text {loss }}=\frac{Z_{0} c}{2 \pi a b} \mathrm{H}\left(k_{0} \sigma_{z}\right)
$$

In case of a rectangular waveguide they are

$$
k_{0}^{2}=f \frac{2}{\delta} \frac{a+b}{a b}, \quad k_{\text {loss }}=\frac{Z_{0} c}{8 a b} \mathrm{H}\left(k_{0} \sigma_{z}\right)
$$

$a$ and $b$ are the semi-axes of the elliptical or half the width of the rectangular waveguide respectively.

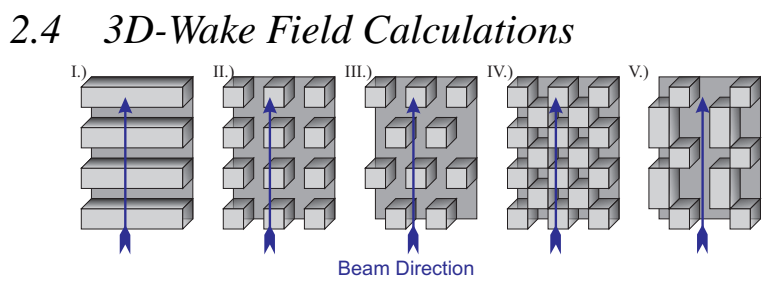

Figure 4: Surface structures of a rectangular waveguide used for 3 dimensional wake field calculation. I.) irises, II.) cubes, III.) shifted cubes IV.) chequered and V.) 2 periods.

In order to check the model many 2-dimensional calculations were performed up to this point of time. Now the results of 3-dimensional calculations are presented to show the validity. Common to all computations is the use of a quadratic waveguide with $2 \mathrm{~mm}$ width, only to verify Eq. 7 waveguides with arbitrary aspect ratio are used. The structure depth as well as the bunch length are $100 \mu \mathrm{m}$. Schematics for the used surface structures can be seen on Fig. 4. Unless otherwise noted, the width of one gap or one iris is $100 \mu \mathrm{m}$ too.

The wake fields show the same sine-like behaviour as in the 2-D case. The calculated loss factors and energy spreads (Fig. 5) principally follow the course of the theoretical values (Fig. 3, turquoise curves in Fig. 5). Using the "2 period structure" (Fig. 4.V) the wake field is partly com2 pensated (Diamonds on Fig. 5). But if the period length becomes shorter in comparison to the bunch length, the wake follows the curve again. 

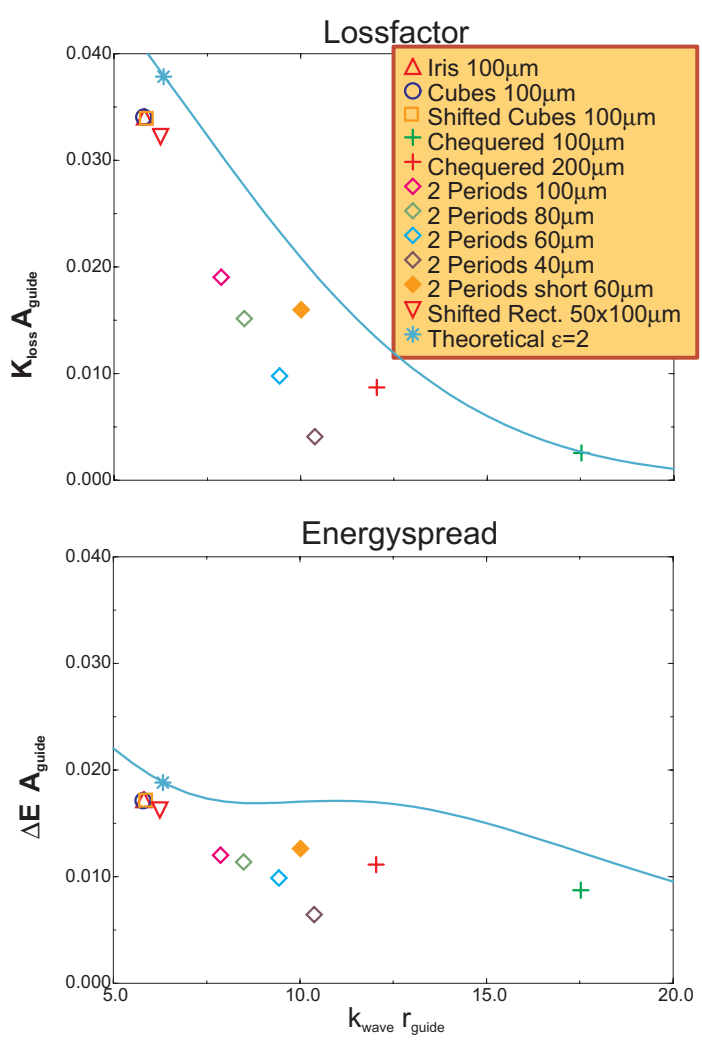

Figure 5: Comparison of loss factor and energy spread of 3D calculations to theoretical values. The star marks the $2 \mathrm{D}$ result for irises in a rectangular waveguide. The curves are a sector of Fig. 3

\section{THE EXPERIMENT}

One possibility to measure the surface roughness wake field effect is the measurement of the energy spread and the energy loss directly with the beam. Another possibility is to guide the rf-fields to an appropriate detector, where the frequency spectrum is measured. However the distinctness of the proof of the surface roughness wake fields does strongly depend on the tube length.

\subsection{Proposal for Measurements at the TTF}

Table 1: Parameters of the TESLA Test Facility beam

\begin{tabular}{||c|c||}
\hline \hline Bunch Length $\sigma_{z}[\mu \mathrm{m}]$ & $\geq 250$ \\
Beam Energy $E_{0}[\mathrm{MeV}]$ & $240-390$ \\
Bunch Charge Q $[\mathrm{nC}]$ & 1 \\
Repetition Rate $[\mathrm{Hz}]$ & 10 \\
Bunch Spacing $[\mu \mathrm{s}]$ & 0.111 \\
Bunches per Bunch Train & $1-7200$ \\
\hline \hline
\end{tabular}

The parameters of the beam available at the TTF are given in Tab. 1. Behind the TTF-FEL undulator is some space to install a surface roughness experiment. Since it will be small in comparison to an undulator length of $100 \mathrm{~m}$ or a length of $12 \mathrm{~km}$ for the TESLA-FEL transfer line, an artificially increased roughness has to be attached to the tubes used for the experiment. The experiment can be set

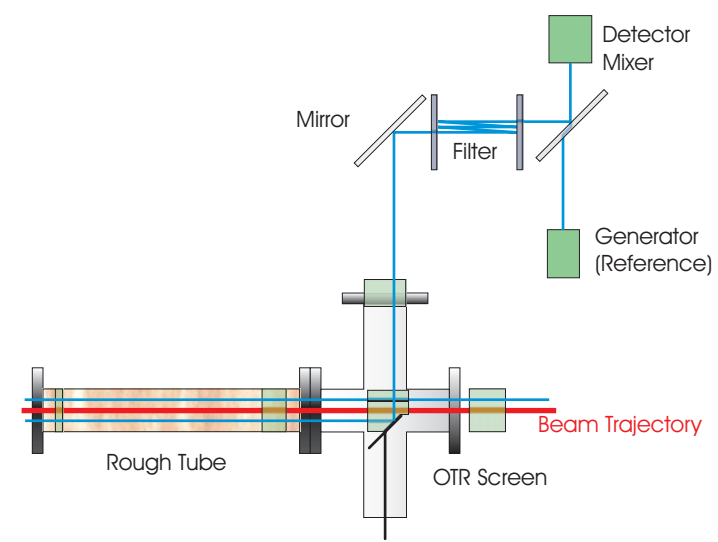

Figure 6: Scheme of the Roughness Wakefield Experiment. The bunch travels through a tube with artificially increased roughness. The rf-pulse, which accompanies the bunch is partially reflected out by an OTR-screen and guide to a detector.

up for a single shot mode. The expected values for the on the beam rf-wake field pulse are given in Tab. 3 .

Table 2: Beam Measurements: loss factor and energy spread per $1 \mathrm{~m}$ rough tube at $240 \mathrm{MeV}$.

\begin{tabular}{|c|c|c||}
\hline \hline Tube & $\begin{array}{c}\mathrm{a}=4 \mathrm{~mm}, \\
\delta=50 \mu \mathrm{m}\end{array}$ & $\begin{array}{c}\mathrm{a}=3.3 \mathrm{~mm}, \\
\delta=75 \mu \mathrm{m}\end{array}$ \\
\hline$k \sigma_{z}$ & 1.118 & 1.005 \\
Loss Factor $\left[\frac{\mathrm{kV}}{\mathrm{m}}\right]$ & 322 & 602 \\
Rel. Energy spread $\frac{\Delta E}{E_{0}}$ & $1.4 \times 10^{-3}$ & $2.05 \times 10^{-3}$ \\
\hline \hline
\end{tabular}

Table 3: RF-wake field pulse per $1 \mathrm{~m}$ rough tube

\begin{tabular}{||c|c|c||}
\hline \hline Tube & $\begin{array}{c}\mathrm{a}=4 \mathrm{~mm}, \\
\delta=50 \mu \mathrm{m}\end{array}$ & $\begin{array}{c}\mathrm{a}=3.3 \mathrm{~mm}, \\
\delta=75 \mu \mathrm{m}\end{array}$ \\
\hline Frequency $[\mathrm{GHz}]$ & 214 & 192 \\
Pulse Duration $\sigma_{e}\left[\frac{\mathrm{ps}}{\mathrm{m}}\right]$ & 65 & 65 \\
Pulse Energy $\left[\frac{\mathrm{mJ}}{\mathrm{m}}\right]$ & 0.32 & 0.6 \\
Pulse Power $[\mathrm{kW}]$ & 4960 & 9260 \\
\hline \hline
\end{tabular}

\section{REFERENCES}

[1] A. Novokhatski and A. Mosnier, 'Wakefields of Short Bunches in the Canal Covered with thin dielectric layer', PAC'97,p. 1444, Vancouver, Canada, May 1997.

[2] A. Novokhatski, M. Timm, and T. Weiland, "The Transverse Wake Fields in the TESLA-FEL Transfer Line," Proceedings of the EPAC-98, pp. 515-517, 1998.

[3] A. Novokhatski, M. Timm, and T. Weiland, "The Surface Roughness Wake Field Effect," Proceedings of the ICAP'98, to be published, 1998.

[4] A. Novokhatski and T. Weiland, "Self-consistent model for the beams in accelerators," Proceedings of the ICAP'98, to be published, 1998. 